\title{
The curious case of elevated thumbnail mercury content
}

\author{
AARON ZIMMERMAN* ${ }^{*}$, JUDITH L. HANNAH ${ }^{1,2}$, HOLLY J. \\ STEIN $^{1,2}$ \\ ${ }^{1}$ AIRIE Program, Colorado State University, Fort Collins, \\ Colorado 80523, USA \\ (*aaron.zimmerman@colostate.edu) \\ ${ }^{2}$ Institute of Geosciences, University of Oslo, Norway
}

Mercury, an infamous and highly studied potentially harmful element (PHE), strikes fear in the general public when concerns of exposure, poisoning, and health impacts are broadcast by the media. Measuring, monitoring, and remediating environmental mercury exposure pathways are critical to allay the public's concern and lessen the health and financial burden caused by mercury exposure.

Measuring mercury in environmental and biological samples is crucial to monitor and eradicate exposure pathways. Thermal decomposition, amalgamation/atomic absorbtion spectoscopy (TDA/AAS) proves the most rapid, most convenient, and lowest cost method for total mercury content measurement. Mercury concentrations for environmental and/or biological samples are produced within minutes for less than $\$ 100 /$ sample.

The AIRIE Program is using a direct mercury analyzer (DMA) for characterizing mercury contents of geological samples. While focusing on geological and environmental samples, curiosity drove us to analyze a few easily accessible biological samples as well, namely, fingernail clippings. Along with hair, blood, and urine, fingernail clippings record mercury exposure. A time series of eight clippings was created to (1) test our ability to analyze biological samples whilst also documenting the accuracy and precision of the instrument, (2) test for any temporal change in fingernail mercury content, and (3) provide a case study for any future biological mercury analyses.

We noted a curious result. Both thumbnails show elevated mercury relative to the other 8 fingernails. Thumbnails average $30 \%$ more mercury than fingernails albeit with a high variance $(0-50 \%$ enrichment). It appears we are the first laboratory to report this observation. The ability of the DMA to statistically differentiate mercury content proves it to be a powerful tool when characterizing mercury budgets and distributions.

The biological mechanism for the disperate mercury content is currently unknown. We report our data as a springboard for (geo)biologists to investigate. We are convinced the DMA and TDA/AAS methodologies will prove vital in understanding this and other mercury-related quandries. 